\title{
TIENDAS TEMPORALES PARA EMPRENDEDORES \\ EN MODA DE GUAYAQUIL
}

\author{
TEMPORARY STORES FOR ENTREPRENEURS \\ IN FASHION GUAYAQUIL
}

\section{CHRISTIAN ROSERO', CECILIA VÉLEZ BARROS², MARÍA EMILIA RUIZ², BEATRIZ LOOR ÁVILA}

\footnotetext{
1 Universidad de Especialidades Espíritu Santo. chrosero@uees.edu.ec

2 Universidad Católica de Santiago de Guayaquil. cecilia.velez@cu.ucsg.edu.ec

3 Universidad de Especialidades Espíritu Santo. maruizpa@uees.edu.ec

4 Universidad Católica de Santiago de Guayaquil. beatriz.loor@cu.ucsg.edu.ec
}

RESUMEN

Las tiendas temporales aparecen en el mercado de moda europeo en el 2003, como una nueva alternativa al marketing tradicional, capaz de lograr un enganche con los consumidores sin exigir mucho presupuesto. La investigación que da pie al artículo, tuvo un enfoque cualitativo. Se realizaron entrevistas a profundidad a emprendedoras en moda en Guayaquil entre los 20 y 35 años de edad y observaciones a una tienda temporal instalada en la ciudad. Se concluyó que los emprendedores en moda están interesados en este concepto y entre sus múltiples ventajas se encuentran: la expectativa a la cual el cliente es expuesto por la marca, la interacción entre el consumidor y los clientes que pertenecen a nuevos mercados.

PALABRAS CLAVE: Tiendas temporales, marketing de experiencias, moda, emprendedor.

\section{ABSTRACT}

Temporary shops appear in the European fashion market in 2003, as a new alternative to traditional marketing different, able to achieve engagement with consumers without requiring much budget. The research was qualitative in nature, depth interviews entrepreneurial fashion in Guayaquil between 20 and 35 years old and watching a temporary tent set up in the city were made. It was concluded that entrepreneurs are interested in fashion and this concept among its many advantages include: the expectation to which the client is exposed by the brand, the interaction between the consumer and the opportunity to test new markets.

KEYWORDS: Temporary stores, experiential marketing, fashion, entrepreneur 
INTRODUCCIÓN

Después de los años cincuenta del pasado siglo, las tiendas generaron un gran impacto en la población, debido a los cambios en las condiciones de vida de los consumidores; como por ejemplo el crecimiento de las ciudades, la aparición de barrios residenciales, etc., dando origen a las tiendas de descuento (outlets), tiendas departamentales y nuevas políticas de precios promocionales (Kim, Jolly, Fairhurst, \& Atkins, 2005).

El surgimiento de las tiendas temporales se ubica en el año 2003, en Gran Bretaña; esparciéndose rápidamente a Estados Unidos y luego a toda Europa para el año 2005. Marcas pioneras como Target, Vacant, Comme des Garçons, entre otras, decidieron abrir tiendas por períodos de tiempo corto (máximos de un año y mínimos de un día, o incluso menos si la mercadería se agotaba rápido). En el 2005 en Milán, Italia, L’Oreal abre su primera tienda "pop up" con el fin de lanzar al mercado un nuevo producto de Lancôme llamado Resurface Peel. Pocos meses después, Levi's abrió en la misma ciudad su tienda temporal por aproximadamente seis meses. A partir de ese momento, el fenómeno se extiende rápidamente en Italia, desde Roma hasta Nápoles, y en pequeños pueblos y villas (Pomodoro, 2013).

Este modelo de negocios permite ofrecer artículos de edición limitada en un ambiente diferente donde los consumidores no sólo compran, sino que además se llevan una experiencia con la marca. Una de sus principales características es que un día están y al siguiente ya no (Julep Beauty Inc., 2013). Utilizan el factor sorpresa y la expectativa para motivar la curiosidad de los consumidores y que al mismo tiempo ellos se conviertan en voceros de la marca a través del boca a boca (Tzortzis, 2004; Gray, 2012).

El éxito de las tiendas temporales se ha visto reflejado en la expansión de este modelo alrededor del mundo, en países y ciudades como: New York, Inglaterra, Berlín, Tokio, Italia, Francia, Escandinavia, Eslovenia, España. Ha llamado la atención de marcas reconocidas mundialmente como Nike, Yves Saint Laurent, JC Penney y Gucci; y otras no tan conocidas como Swim Spot Holdings LLC. Incluso marcas que se alejan del sector de la moda como Toys "R" Us, eBay, The Original Factory Shop y la revista Monocle Magazine ya han hecho de las tiendas temporales una parte importante de sus estrategias de comunicación y de marketing (Tzortzis, 2004; Endelson, 2009; Hamanaka, 2012).

En Milán, se ha dado origen a una nueva asociación dedicada únicamente a tiendas y espacios temporales llamada 'Assotemporary'. Esta asociación, conformada por representantes de marcas de moda de varias ciudades italianas, fue creada en mayo del 2008 y ha logrado desarrollar varios proyectos a través de estudios de lugares con mayor tráfico de personas, análisis de factores importantes para la elección de lugares estratégicos, número de habitantes en la zona, actividades comerciales del sector, etc. La asociación ha reportado la clausura de alrededor de 500 tiendas convencionales en Milán sólo en el 2008, y la apertura de 50 tiendas temporales aproximadamente en el mismo período (Surchi, 2010; Assotemporary, 2012).

Ryu (2011) manifiesta que la implementación y operación de tiendas pop -up son una estrategia efectiva de retail que logra adaptarse a los intereses de los consumidores hedonistas, que hoy en día "buscan emociones a gran velocidad para poder consumirlas" (García, 2008, p.79). Las tiendas temporales satisfacen esa demanda de incentivos inmediatos, a la vez que cubren las necesidades de la marca por sobresalir dentro de un mercado saturado (García, 2007).

En Ecuador, y más específicamente en la ciudad de Guayaquil no se ha visto una fuerte evidencia del uso de tiendas temporales, a excepción de la tienda Magnum ubicada en el patio de comidas de Mall del Sol, inaugurada el 14 de febrero y desmontada el 26 de marzo de 2014. Es la primera tienda en Guayaquil que cumple las características de un pop up, pero pocos conocen el concepto. Esto constituye una desventaja para las marcas ecuatorianas que desean emprender y no tienen las herramientas o recursos para realizar pruebas de mercado, encontrar nichos, y crear conciencia de marca con poco presupuesto. No se ha desarrollado un modelo de negocios bajo el esquema de tiendas temporales como herramienta de marketing en Guayaquil; razón por la cual, el sector de la moda está perdiendo oportunidades de crecimiento.

Tomando en cuenta la situación previamente mencionada, en el presente estudio se planteó el objetivo de conocer cómo desarrollar un concepto de tiendas temporales adaptado a las necesidades del mercado de un producto o marca. Para este propósito se escogió como análisis el mercado de moda de la ciudad de Guayaquil.

Se realizó un estudio de tipo cualitativo bajo enfoque de las experiencias de los actores clave de dicho mercado y se esquematizó de la siguiente manera: El marco conceptual que realiza una revisión literaria sobre tiendas temporales, el aspecto metodológico que explica 
cómo se cumplieron los objetivos establecidos, y finalmente los resultados y conclusiones basadas en la investigación realizada.

\section{MARCO CONCEPTUAL LAS TIENDAS TEMPORALES}

Los centros comerciales están innovando continuamente para responder a las demandas del mercado (Fenley, 2003). Esto se ve reflejado en sus nuevos formatos que buscan conectarse con el consumidor de una manera diferente como es el caso de los centros comerciales al aire libre, o los de una sola parada (one-stop shops), las plazas, etc. (Niehm, Fiore, Jeong, \& Kim, 2007). Las tiendas temporales, también conocidas como tiendas "pop up" (Surchi, 2010), son un nuevo formato del marketing de experiencias, creado para lograr un enganche con los consumidores (Niehm, Fiore, Jeong, \& Kim, 2007; Pine \& Cilmore, 2011). El término "tienda temporal" ha sido creado para describir una tienda de alguna marca en particular que ha sido abierta por un período corto de tiempo, generalmente en un lugar representativo de la ciudad, que atrae rápidamente al público en torno a un evento antes de desaparecer completamente, transportarse a otra localidad, o transformarse en algo totalmente diferente (Caridà, Colurcio, Melia, \& Russo, 2012).

La principal característica de las tiendas pop up es su corto tiempo de vida. Convirtiéndose en eventos irrepetibles, caracterizadas por una participación multisensorial entre el consumidor y la marca. Por esta razón, suelen ser catalogadas como nueva tendencia en este sector en las estrategias de marketing (Niehm, Fiore, Jeong, \& Kim, 2007; Surchi, 2010). En cuanto a su segunda función, las tiendas temporales actúan como una herramienta para realizar pruebas de mercado. A través de ellas se puede identificar el potencial de ventas que tiene un nuevo mercado o qué tanta acogida tiene una marca o producto en un determinado sector antes de realizar la inversión que requiere la compra e inauguración de una tienda permanente (Surchi, 2010). Por otro lado, las marcas nuevas, que se encuentran en su etapa de introducción o crecimiento, utilizan principalmente la función investigativa de las tiendas temporales. Con esto, se trata de conseguir mayor visibilidad y probar el grado de acogida que tiene el mercado hacia sus productos, evaluar cómo los consumidores responden a la marca en localidades específicas, y a partir de eso, tener las bases para desarrollar una estrategia de marketing adecuada, sin la necesidad de realizar una fuerte inversión (Pomodoro, 2013; Surchi, 2010).
Las metas y los objetivos trazados por una empresa bajo el contexto de tiendas temporales no son los de generar un aumento significativo de las ventas, sino principalmente comunicar la marca; las ventas son una función secundaria (Marciniak \& Budnarowska, 2009). Varios autores, han asignado a este fenómeno objetivos estratégicos; entre ellos: crear eventos y acontecimientos, despertar emociones, iniciar nuevas tendencias, probar una marca o producto, fomentar las compras por impulso, y proporcionar presencia física para empresas virtuales (Caridà et al., 2012).

Se debe dar especial énfasis en la novedad y exclusividad que debe brindar esta herramienta a la marca que la utilice. Como explica Pomodoro (2013), el mercado está tan saturado de marcas, que cada una necesita crear algo que la diferencie y la haga destacar. Las tiendas temporales logran ese efecto "sorpresa" en el consumidor a través de su corto tiempo de vida. El principal estímulo que causa en el consumidor es el sentimiento de "ansiedad" por quedar excluido de un evento, de perderse algo único, fugaz e irrepetible (Catalano \& Zorzetto, 2010).

El producto que se ofrece en una tienda temporal debe ser igual de exclusivo que la tienda. Muchas empresas se inclinan hacia la venta de productos y marcas de edición limitada (Pomodoro, 2013).

El consumo ha empezado a verse como un constante flujo de fantasías, sentimientos y diversión, incluido lo que se conoce como 'experiencia' (Holbrook \& Hirschman, 1982). Esta última, es fundamental en una estrategia basada en tiendas temporales y se debe crear en cada punto de contacto entre el consumidor y la maca; desde la misma tienda hasta la comunicación a través de la publicidad, redes sociales, página web, etc. (Niehm, Fiore, Jeong, \& Kim, 2007). La experiencia no es una herramienta tangible que se puede vender o transferir de la empresa al consumidor. Al contrario, es co-creada a través de la participación del consumidor con un ambiente sensorial y emocional (Pine \& Gilmore, 2011; Caridà et al., 2012).

Al lograr este tipo de experiencia más completa se generan comentarios positivos en torno a la marca a través del boca a boca, se fortalece la imagen y recordación de la marca, y mejora su posicionamiento en la mente del consumidor (Woodside \& Walser, 2007; Marciniak \& Budnarowska, 2009). Las marcas que crean repetidas experiencias que permiten al consumidor interactuar con ella, como es el caso de la tiendas 
pop up, tienen grandes posibilidades de fidelizar a sus clientes y lograr una fuerte ventaja competitiva (Ponsonby-McCabe \& Boyle, 2006).

Según Levinson (2007), esta cualidad de brindar experiencias, calificaría a las tiendas temporales como una poderosa herramienta del marketing de guerrilla. El marketing de guerrilla es un conjunto de estrategias y técnicas de marketing ejecutadas por medios no convencionales, y que consiguen su objetivo mediante el ingenio y la creatividad, en vez de ser mediante una alta inversión en espacios publicitarios. De hecho, las tiendas temporales tienden a utilizar herramientas poco convencionales y de bajo costo (Pomodoro, 2013; Qader, 2013). Es así como muchos minoristas han decidido utilizar el marketing de guerrilla para complementar sus técnicas tradicionales de comunicación y poder responder a los cambios en las preferencias de los consumidores (Collins, 2004). Esto, conduce a las tiendas a dejar de ser tan sólo un punto de venta, para convertirse en un lugar donde marca y consumidor construyen y desarrollan su relación y donde ocurre un fuerte intercambio emocional (Niehm et al., 2007; Caridà et al., 2012)

La ubicación geográfica de una tienda es un aspecto estratégico a considerar, ya que impulsa el acceso de los consumidores a las experiencias y asegura que el evento temporal sea altamente visible al público (Caridà et al., 2012). Pomodoro (2013), indica que el lugar escogido para colocar la tienda debe ser innovador, es decir, debe ir más allá de las formas tradicionales y sugiere galerías de arte, contenedores de barcos, etc. Marcas como Uniqlo y L'Oréal por ejemplo, escogieron las estaciones del subterráneo en Union Square y Bryant Park, Estados Unidos, respectivamente para introducir nuevos conceptos (Moin, 2013).

Las tiendas temporales siguen la misma tendencia que las tiendas insignia, ya que buscan áreas con mayor actividad comercial como los centros de la ciudad, o cualquier zona que sea popular entre los consumidores. Dentro de una estrategia basada en tiendas temporales, la localización se convierte en un indicador de tendencias para la marca (Marciniak \& Budnarowska, 2009). Por ello, Soho, Nueva York se convirtió en la zona predilecta para los minoritas de moda temporales (Pomodoro, 2013).

\section{METODOLOGÍA}

La investigación siguió los cánones de una investigación cualitativa, donde se utilizaron dos técnicas, la entrevista a profundidad y la observación en sitio. La población de estudio fueron mujeres diseñadoras de prendas de vestir, trajes de baño, calzado y accesorios, entre los 20 y 35 años de edad que tienen una marca propia de moda en la ciudad de Guayaquil. La muestra seleccionada se subdividió en aquellas personas con tienda propia o showroom, y las que no tienen espacio físico para mostrar la mercadería. Se realizaron 6 entrevistas a profundidad a dueñas en su mayoría mujeres diseñadoras de moda con tendencias prêt - à - porter, es decir que no tienen una producción en masa, sino algo más pequeño, personali, de tiendas y 6 informales calzado y trabajado a mano; $y$ otras pocas de tendencia prêt - à -couture que diseñan con un poco más de alta costura y elaboran diseños únicos.

Fueron entrevistados emprendedores en moda a través de una guía de preguntas, con el objetivo de conocer los modelos que están utilizando actualmente para promover sus marcas, sus retos, y efectividad de su comunicación. De igual forma, se indagó sobre el grado de conocimiento del concepto de tiendas temporales, oportunidades que le ven al modelo, factores relevantes a considerar para su implementación y recomendaciones para adaptarlo al mercado Guayaquileño.

Se realizó la observación a través de una guía estructurada a la tienda Magnum ubicada en Mall del Sol, para obtener información relacionada al diseño, tamaño, atención al cliente, merchandising, decoración, etc. Se realizó además una breve entrevista a uno de los empleados de la tienda sobre el funcionamiento y logros alcanzados en Guayaquil.

Dentro del diseño exploratorio, se aplicó además la técnica de entrevistas en profundidad, con el objetivo de recabar información acerca de factores relevantes para el desarrollo de tiendas temporales, beneficios y principales funciones. Se realizaron entrevistas a profundidad a emprendedores en moda, para lo cual se utilizó el método bola de nieve es decir, a través de referencias se pidió a los entrevistados que sugieran otras personas que trabajen en el mismo sector de moda para realizarles la entrevista. Algunas de las personas entrevistadas fueron encontradas a través de redes sociales como Facebook o Instagram, o por contactos personales.

ANÁLISIS DE RESULTADOS

Para el análisis e interpretación de los datos cualitativos obtenidos de las entrevistas se clasificó la información en tres categorías diferentes para responder a los principales objetivos planteados en la investigación. La primera corresponde al conocimiento general y 
situación actual de la marca; la segunda trata el conocimiento del concepto de tiendas temporales por los emprendedores en moda y la proposición de un concepto de tienda adecuado para los negocios de moda en Guayaquil. La tercera comprende la elección de un modelo de tienda adecuado para Guayaquil tomando en cuenta los principales elementos del marketing mix (producto, precio, plaza y promoción), más un quinto elemento fundamental para las tiendas temporales: tiempo.

La primera categoría abarca principalmente las técnicas de comunicación aplicadas por los emprendedores en moda para llegar a su grupo objetivo; las principales dificultades que experimentan en este aspecto dentro del mercado guayaquileño; y en el caso de poseer una tienda, los retos y aspectos importantes a considerar al abrir un local.

Las herramientas de comunicación más utilizadas por las emprendedoras en moda son las redes sociales y el boca a boca. Luego están las ventas online o páginas web, participación en ferias y unas pocas también han logrado dar a conocer su marca a través de entrevistas por televisión. Estas herramientas han sido útiles. Sin embargo, uno de los principales retos que encuentran en este proceso es la necesidad de constante innovación, y la poca valoración y apertura de los consumidores guayaquileños hacia nuevos diseñadores, especialmente nacionales. Adicionalmente, ya que su estrategia se basa principalmente en redes sociales, está el reto de conseguir más seguidores y lograr la compra definitiva del producto. "Muchas veces una prenda logra muchos 'likes', pero de ahí no pasa" (Almachi, 2014).

Muchas de las emprendedoras son dueñas de una tienda, otras tienen sus talleres o showroom para exhibir sus prendas y algunas están en proceso de convertirlos en tiendas. La mayoría coincide en que los retos que enfrenta una tienda son: encontrar la ubicación idónea y la seguridad de insertar sus productos al mercado. Los permisos para operar y el mantenimiento del local, los costos de alquiler y de operaciones. Que las ventas no logren cubrir esos gastos es una de las mayores preocupaciones de las emprendedoras. Una de ellas comentó que lo más difícil es "encontrar un local que se ajuste exactamente a lo que uno necesita [...] a veces uno pierde los objetivos de vista, en la administración de un local, consumiendo tiempo y recursos" (Rodríguez, 2014).

La segunda categoría se enfoca en el concepto adecuado de tiendas temporales para
Guayaquil. Lo cual abarca las oportunidades, ventajas y desventajas, formas de adaptarlo a este mercado y recomendaciones para su correcta implementación por parte de los emprendedores en moda de la ciudad.

De las entrevistas a profundidad mencionamos cinco personas que tienen conocimiento de lo que son las tiendas pop up. Conocen que son muy populares en Estados Unidos y Europa, que se ubican en zonas muy concurridas, y que no hay evidencia de ellas en Guayaquil. Lo más cercano a este concepto que ellas han visto aquí, es el mercadillo o algo más popular como las ferias del jean por ser "temporales", pero no terminan de cumplir con las características de una pop up.

En general, las entrevistadas opinan que las tiendas pop up como herramienta de marketing son una idea innovadora. Muchas se muestran entusiasmadas por la idea de probarlas con sus propios negocios afirmando que son un modelo realmente viable y fuera de lo tradicional, con muchas oportunidades en especial para líneas comerciales como las de ellas. Indican que es una herramienta ideal para marcas que quieren entrar al mercado y no tienen suficientes recursos para adquirir un local permanente.

Entre las principales ventajas que se pudieron delimitar entorno al uso de tiendas temporales para los negocios de moda, se encuentran las siguientes: a) el hecho de ser temporales permite probar con un mercado más grande sólo por un tiempo; b) permite ahorrar el costo de los locales y abaratar costos de operación; c) concede a marcas pequeñas la oportunidad de ofrecer sus productos y diseños a los consumidores; d) otorgan más oportunidades en temporadas de alta demanda; e) requieren un monto de inversión bajo; f) permiten posicionar la marca, y especialmente que los consumidores la vean como una marca innovadora; g) dan la oportunidad de probar el mercado sin comprometerse a un contrato de largo plazo; h) ofrecen la sensación de exclusividad porque el público sabe que es un evento único y se lo pueden perder, por lo tanto, crean esa sensación de urgencia, de asistir y comprar su producto en los consumidores; i) los costos fijos son mucho más bajos que los de un negocio tradicional; j) se mantiene al cliente a la expectativa de lo nuevo que la marca puede hacer; k) no se necesita mantener un stock elevado y se reduce el personal; l) mayor flexibilidad para organizar eventos, y probar con nuevos consumidores, nuevos targets; m) facilita la realización de pruebas de mercado; por ejemplo para lanzamientos de nuevos productos, líneas, 
o colecciones, en lugar de hacer un grupo focal tienen la tienda pop up; n) a diferencia de un mercadillo, la tienda se enfoca únicamente en su marca; y ñ) estimulan la interacción entre el consumidor y la marca.

A pesar de considerar a las tiendas temporales como una excelente estrategia de marketing, algunas de las emprendedoras entrevistadas no saben si en Ecuador logren la misma acogida que en otros países más avanzados donde se han implementado. Una de las mayores preocupaciones entre las diseñadoras es la poca garantía en el mercado para un emprendedor que hay en el país, lo cual reduce el número de lugares para colocar una tienda pop up. Otro aspecto importante a considerar, es tener el tiempo correcto para preparar la tienda y darla a conocer al público. Por lo tanto, les preocupa la poca asistencia e interés por parte de los consumidores hacia la tienda y que por ello no se logren los objetivos deseados y su implementación no justifique la inversión. Adicionalmente, opinan que es una desventaja para las tiendas temporales, el hecho de que si se logran los objetivos deseados y si funciona correctamente no se pueda convertir en una tienda permanente.

Tomando en cuenta los aspectos positivos y negativos delimitados, las emprendedoras en moda ofrecieron una serie de puntos importantes que se deben investigar antes de colocar una tienda temporal en Guayaquil: a) como punto primordial y básico está la selección de la ubicación de la tienda; b) la seguridad y el clima son factores muy influyentes ya que no permiten poner una pop up donde sea; c) el concepto de comunicación; d) el comportamiento del consumidor y comportamiento de compra: gustos, costumbres, dónde compra, qué compra, cómo compra, qué espera de un evento como este, etc.; e) el valor agregado que se le puede dar al evento para lograr impacto y hacerlo viral; f) el personal necesario; g) la publicidad que amerita; h) visual merchandising para crear un mejor impacto; i) los permisos necesarios; j) el stock necesario; k) las formas de pago; y l) los costos, porque aunque será efímera, debe mantener la buena imagen de la marca.

Es importante, delimitar el lugar correcto; para esto es necesario tener claramente definido a quién se está dirigiendo la marca. Muchas entrevistadas sugirieron un local comercial en un mall concurrido, como la mejor opción para colocar una pop up por razones de seguridad y de comodidad para los consumidores. Indican que la actitud "novelera" de los consumidores gua- yaquileños es un factor que pueden aprovechar las tiendas temporales. Sin embargo, es necesario trabajar en la percepción del cliente hacia este nuevo modelo, y que entienda claramente de lo que se trata. Para no dañar la imagen de la marca, una vez que la tienda desaparezca, es importante que el consumidor comprenda que la tienda es efímera. Por lo tanto, debe existir una buena comunicación por parte la marca en cuanto al tiempo que estará abierta y continuar en contacto con el consumidor una vez terminado este período.

En vista de que las tiendas temporales están diseñadas para ser utilizadas por cualquier marca, sea grande o pequeña, las entrevistadas recomiendan a las marcas pequeñas o poco conocidas utilizar las tiendas para promocionar su última colección. Sugieren además, que las marcas conocidas se pueden arriesgar más y aprovechar la tienda para sacar una colección única o producto único.

Las diseñadoras indicaron que está en boga el uso del marketing aplicado a los sentidos y que va de la mano con este modelo de tiendas temporales emprendedoras, por lo tanto se debe aprovechar para crear experiencias con la marca. También consideran importante aprovechar las temporadas de alta demanda del producto para abrir una tienda temporal como por ejemplo la temporada playera en el caso de las diseñadoras de trajes de baño. Otras, sugieren la creación de pequeños eventos alrededor de fechas importantes para el país, ciudad o celebraciones mundiales. Esta forma, es llamada "dayketing", que se refiere al marketing por fechas, es decir, aprovechar días especiales para dar algo especial a los clientes: descuentos, eventos, regalos, sorpresas, sorteos, concursos, lo que vaya mejor con la fecha.

Finalmente, no puede faltar el apoyo de una fuerte estrategia publicitaria, una buena decoración que vaya acorde al concepto e imagen de la marca y tener lo último en tendencias de moda.

La tercera categoría del análisis se refiere al modelo más adecuado para un negocio de moda en Guayaquil. Los resultados obtenidos en esta parte de la entrevista fueron muy variables ya que iban más acorde a los gustos y estilos de cada una de las entrevistadas. Tomando en cuenta la variable "producto" por ejemplo, muchas coincidieron en que el tamaño de la tienda debería ser pequeño. Sin embargo, hablando de medidas algunas prefirieron el tamaño de un contenedor pequeño, unas de 3 x 3 y otras hasta de $10 \times 10$ metros. Al preguntar sobre el diseño 
de la tienda cada cual escogió un estilo ajustado a sus diseños; desde colores neutrales, con temática, minimalistas, ambientadas según el evento, hasta ecológicas/industriales.

Algunas entrevistadas, aportaron con algo diferente sobre el tipo de productos que ofrecerían en la tienda, sugiriendo la creación de una tendencia o una "colección cápsula1", ideas que van muy de la mano con el objetivo de una tienda pop up.

En cuanto al lugar más adecuado para colocar un tienda pop up, sobresalieron las zonas de Samborondón y Urdesa. Un grupo menor mencionó Kennedy y Ceibos, pero la gran mayoría indicó que lo mejor es ubicarla dentro de un mall en cualquiera de estas zonas.

El monto de inversión que las entrevistadas estarían dispuestas a dar para la implementación de una tienda temporal fue muy variable. El menor fue de $\$ 200$ y el mayor de $\$ 20,000$. La inversión debería cubrir los gastos que ellas consideraron estrictamente necesarios como: permisos, materiales para decoración, prendas, modulares para colocar las prendas, maniquís, comodidad (aire acondicionado si no se coloca en un mall), publicidad, diseño, movilización, iluminación, seguridad, personal, alquiler, y alícuotas.

Entre las actividades sugeridas para realizar en una tienda pop up, más allá de la venta de la mercadería está: customizar las prendas, outlets por temporadas, sorteos, desfiles, eventos por fechas especiales, lanzamientos, promociones, asesoría de imagen, etc.

El tiempo de duración para una tienda temporal que las entrevistadas consideran prudente y necesario para cumplir con los objetivos planteados, fue variable de una diseñadora a otra. Algunas afirmaron que de 1 a 3 días, mientras que la gran mayoría señalaban entre 3 y 4 meses.

De la observación realizada a la tienda temporal de Magnum se pudieron obtener datos relacionados al diseño de una pop up en Guayaquil. Las características que se hallaron en esta fueron, el uso de materiales como MDFutilizado en el recubrimiento de toda la decoración, acabados de laca blanca, material metálico dorado, mucha iluminación y un tamaño aproximado de 4 x 4 metros. Parte de la decoración de la tienda incluía además, urnas iluminadas con elementos elaborados de diferentes materiales que simulaban un helado Magnum. Adicionalmente, colocaron una paleta con instrucciones

Colección pequeña y limitada. También puede servir como abreboca de una nueva colección. para los consumidores sobre el producto que estaban ofreciendo y los pasos a seguir dentro de la tienda y posterior a la prueba del producto. El precio del producto era de $\$ 2,50$.

El personal estaba uniformado con los colores del producto y un logo que decía "Magnum Ecuador”. Compuesto por cinco personas: una en caja, dos atendiendo a los clientes, dos manteniendo el control y vigilancia debido a la gran concurrencia; y adicionalmente, una impulsadora que de vez en cuando realizaba encuestas a los consumidores sobre los sabores de su preferencia y opiniones en general sobre la tienda y la marca. Las personas hacían fila durante 40 minutos aproximadamente para probar el producto.

La breve entrevista a uno de los empleados de la tienda también arrojó datos muy interesantes sobre su funcionamiento y éxito en la ciudad de Guayaquil. En primer lugar, la tienda tuvo gran acogida en el mercado guayaquileño debido a su actitud "novelera". Atendían aproximadamente a 900 personas diarias. Indicaron que la tienda se quedaba hasta finales de marzo 2016, siendo el 26, el último día en Guayaquil. En el futuro se trasladarán a Quito, después de darle el debido mantenimiento a la tienda.

Asistieron personas provenientes de otras provincias, a quienes se les solicitaron que lleven sus tiendas a sus localidades, por este motivo van a tratar en Quito. Sin embargo, ellos no están seguros de un éxito tan grande como en Guayaquil por la actitud de los consumidores y el clima. Los ejecutivos de Unilever fueron la mañana del último día de funcionamiento de la tienda con el fin de felicitar a los empleados, informándoles de que el éxito logrado estuvo al mismo nivel que Londres, Singapur, Estados Unidos, etc. También están pensando en un posible regreso, luego de revisar los datos de los estudios aplicados para realizar las mejoras necesarias. Los objetivos de la tienda fueron mejorar el posicionamiento de la marca y realizar pruebas de mercado.

\section{CONCLUSIONES}

Para la apertura de tiendas temporales en Guayaquil el reto más grande es el espacio. Estamos hablando de un lugar "altamente visible al público" o zonas "populares". Lo más recomendable sería un centro comercial, como Magnum lo hizo, pero estamos hablando de una marca conocida y con mucho presupuesto en comparación a los emprendedores en moda. La necesidad más grande de Magnum era simplemente innovar y la pop up 
resultó muy bien debido a la novedad de la tienda y al sentimiento de urgencia que creó en los consumidores apoyándose en las redes sociales.

El sentimiento de ansiedad en los consumidores de asistir a un evento único e irrepetible permitió que el proyecto fuera un éxito, que se convirtiera en algo viral. Para un "come back" sería muy exitoso ya que muchos se quedaron sin asistir a pesar de querer hacerlo. Ahora, hablando de una diseñadora joven, la situación es diferente. Dada la cultura en el Ecuador, y Guayaquil específicamente, lo único que conseguirá un microempresario dispuesto a invertir $\$ 1000$ o menos en su marca será un evento pequeño en un parque como Urdesa, o a lo mucho el Mercadito si tiene los contactos, pero bajo ese contexto ya no sería una pop up.

Debido a que la definición de tiendas pop up no tiene bases ni es conocida en Guayaquil, es importante educar al consumidor en base a este tema; de manera que cambie su percepción entorno a las tiendas pop up y no las asocie con los vendedores informales, mercadillos y plazas de ventas que tanto se ven en la ciudad. Es un concepto que el consumidor ya tiene formado en su conciencia, por lo tanto, para su correcto funcionamiento se debe crear una estrategia apoyada en redes sociales y publicidad para informar y educar al consumidor como lo hizo la tienda Magnum.

Una solución es la creación de asociaciones como se hizo en Europa. Varios microempresarios que vendan productos similares o compatibles pueden agruparse para realizar un evento en un lugar conocido, de altura, y poder ofrecer sus productos. De esta manera, se pueden financiar más fácilmente los gastos de incursión e incluso promocionar distintas marcas en un solo nivel, en una sola pop up con distintos ambientes. Así por impulsos, el cliente se siente motivado a comprar un producto y sus complementos.

Otra solución es la creación de agencias dedicadas a desarrollar conceptos, estudios de mercado y encontrar las localidades adecuadas a las necesidades de la marca, de la misma manera como se han desarrollado en otros países. Esto permitiría que marcas, tanto grandes como pequeñas, tengan la oportunidad de lograr sus objetivos con el presupuesto disponible.

En el presente estudio se identificaron los posibles usuarios del modelo tienda temporales emprendedoras, quienes tienen cierto conocimiento y están dispuestos a implementarlo señalando que tiene múltiples ventajas y tomando en cuenta los riesgos que presenta. De igual for- ma, enfatizando en la necesidad de una buena investigación del terreno y del mercado, previo a su implementación. Se recomienda que se realicen nuevos estudios que profundicen en las percepciones del mercado, en los consumidores y su grado de aceptación hacia este nuevo modelo, que por el momento es prácticamente desconocido para los consumidores ecuatorianos. Adicionalmente, se sugiere mayor investigación a profundidad sobre el fenómeno de las tiendas temporales y su impacto en los negocios en etapa de crecimiento y en marcas ya desarrolladas.

\section{REFERENCIAS BIBLIOGRÁFICAS}

Diccionario LID de Marketing Directo e Interactivo. (s.f.). Marketing Directo.com. Obtenido de Marketing Directo.com: http://http://www. marketingdirecto.com/

Assotemporary. (2012). Obtenido de Assotemporary: http://www.assotemporary.it

Ecuadormoda. (2 de Enero de 2013). Obtenido de Ecuadormoda: http://www.ecuadormoda.com

Entrepreneur. (2013). Obtenido de Entrepreneur: http://www.entrepreneur.com/

Almachi, N. (18 de Marzo de 2014). Las tiendas temporales como herramienta de marketing para emprendedores en moda en Cuayaquil. (M. E. Ruiz, Entrevistador) Guayaquil, Guayas, Ecuador.

Arata, A. (2013). AlexandraArata. Obtenido de AlexandraArata: http://www.alexarata.com/

Azuero, M. A. (2012). Guía Académica- Las preguntas más frecuentes sobre marketing. Norma.

Bailey, R. (31 de Mayo de 2013). How do pop-up shops work? . How do pop-up shops work? (S. Thom, Entrevistador) Summituk.

Caridà, A., Colurcio, M., Melia, M., \& Russo, T. (2012). Store experience and co-creation: the case of temporary shop. International Journal of Retail \& Distribution Management, 40(1), 21-40.

Catalano, F., \& Zorzetto, F. (2010). Temporary Store. La Strategia Dell'effimero. Franco Angeli.

Collins, C. (2 de Octubre de 2004). "You Want It? You Got It - Instantly. Retailers Have Gone Beyond Traditional Stores, Catalogs, and Websites. Now They Offer Opportunities 24/7. The Christian Science Monitor.

Davis, K. L. (15 de Noviembre de 2013). Jingle bell pop: Retailers to set up shop in downtown Tulsa. The Oklahoma City Journal Record.

Davis, K. L. (10 de Enero de 2014). Tulsa's Deco District drops pop shops program. The Oklahoma City Journal Record.

Di Palma Wright, V. (2011). How to Run a Pop-Up Store. How to Run a Pop-Up Store. (Katie, Entrevistador) Fashion Entrepreneur Report. 
Endelson, S. (19 de Mayo de 2009). Pop-Ups Offer Retailers Multiple Benefits. WWD: Women's Wear Daily, 197.

Fenley, G. (1 de Abril de 2003). Reinventing the mall: A buzz of renovation and the rise of open-air town centers mark a new era in shopping centers. (Retail at a Crossroads). Display $\mathrm{a}$ Design Ideas.

Ferguson, R. (2008). Word of mouth and viral marketing: taking the temperature of the hottest trends in marketing. Journal of Consumer Marketing, 179-182.

García. (2007). Pomodoro.

García. (2008). BOB. La nueva publicidad del S. XXI. Questiones Publicitarias, 1(13), 79 - 83.

García, C. (2007). Bob. Madrid: Zapping/M\&C Saatchi.

Gordon, K. T. (2004). A "hands-on" approach to marketing your product could be just the thing to win customers. Entrepreneur Magazine, 74-75.

Gray, B. (5 de Diciembre de 2012). On 10th Anniversary of First NYC Pop-Up, Retailers Look Back. New York Observer.

Hamanaka, K. (12 de Marzo de 2012). Raj Retail: From 'Pop-Up' to Permanent. Orange County Business Journal, 3;46.

Hays, C. L. (7 de Diciembre de 2004). Stores That Pop Up and Go Away, on Purpose. The New York Times.

Hoch, S. J., \& Loewenstein, G. F. (1991). TimeInconsistent Preferences and Consumer Self-Control. Journal of Consumer Research, 17(4), 492-507.

Holbrook, M. B., \& Hirschman, E. C. (1982). The experiential aspects of consumption: consumer fantasies, feelings, and fun. The Journal of Consumer Research, 132-140.

Jack Morton Worldwide. (2013). Jack Morton Worldwide. Recuperado el 11 de Octubre de 2013, de Jack Morton Worldwide: http://www.jackmorton.com

Julep Beauty Inc. (12 de Abril de 2013). Innovative Omni Channel Beauty Brand Julep Opens NYC Pop-Up Store. Business Wire.

Kim, Y.-K., Jolly, L., Fairhurst, A., \& Atkins, K. (2005). Mixed-Use Development: Creating a Model of Key Success Factors. Journal of Shopping Center Research, 12(1), 53-75.

Kotler, P. (2005). Las preguntas más frecuentes sobre marketing. Bogotá: Norma.

Kozinets, R. V., Sherry, J. F., DeBerry-Spence, B., Duhachek, A., Nuttavuthisit, K., \& Storm, D. (2002). Themed flagship brand stores in the new millennium: theory, practice, prospects. Journal of Retailing, 78, 17-29.

Levinson, J. C. (2007). Guerrilla Marketing: Easy and Inexpensive Strategies for Making Big Profits from Your
SmallBusiness (Cuarta ed.). New York: Houghton Mifflin Company.

Marciniak, R., \& Budnarowska, C. (2009). Marketing approaches to pop up stores: exploration of social networking.

Martínez-Ribes, L. (2013). Innovación en retail. Marketing más Ventas(290), 74-82.

Moin, D. (26 de Noviembre de 2013). Is This Your Stop? It's Time To Shop. WWD: Women's Wear Daily, 206.

Moral Moral, M., \& Fernández Alles, M. (2012). Nuevas tendencias del marketing: El marketing experiencial. ENTELEQUIA(14), 237-251.

Niehm, L. S., Fiore, A. M., Jeong, M., \& Kim, H.-J. (2007). Pop-up retail's acceptability as an innovative business strategy and enhancer of the consumer shopping experience. Journal of Shopping Center Research, 13(2).

Palmer, T. (26 de Enero de 2002). Sensing a winner: touchy-feely marketing is being employed by fmcg companies as they seek alternatives to the traditional marketing media to give key consumers a comprehensive 'brand experience'. Grocer.

Pine, B. J., \& Gilmore, J. H. (2011). The Experience Economy. Estados Unidos.

Pomodoro, S. (2013). Temporary retail in fashion system: an explorative study. Journal of Fashion Marketing and Management, 17(3), 341-352.

Ponsonby-McCabe, S., \& Boyle, E. (2006). Understanding brands as experiential spaces:. Journal of Strategic Marketing axiological implications for marketing strategists, 175-189.

PRNewswire. (5 de Noviembre de 2005). PRNewswire. Obtenido de PRNewswire: http://www. prnewswire.com

Qader, I. K. (2013). The Evolution of Experiential Marketing: Effects of Brand Experience among the Millennial Generation. International Journal of Academic Research in Business and Social Sciences, 3(7), 331-340.

Rodríguez Coltro, B. (18 de Marzo de 2014). Las tiendas temporales como herramienta de marketing para emprendedores en moda en Guayaquil. (M. E. Ruiz, Entrevistador)

Ryu, J. S. (2011). Consumer Attitudes and Shopping Intentions toward Pop-up Fashion Stores. Journal of Global Fashion Marketing, 2(3), 139-147.

Surchi, M. (2010). The temporary store: a new marketing tool for fashion brands. Journal of Fashion Marketing and Management, 15, 257-270.

Tzortzis, A. (25 de Octubre de 2004). Pop-up stores: here today, gone tomorrow. The New York Times.

Woodside, A. G., \& Walser, M. G. (Enero de 2007). Building strong brands in retailing. Journal of Business Research, 1-10. 